\title{
La chanson, « arme » révolutionnaire et chambre d'écho de la société en Auvergne
}

\section{Frédéric Derne}

\section{(2) OpenEdition \\ 1 Journals}

\section{Édition électronique}

URL : https://journals.openedition.org/ahrf/1608

DOI : 10.4000/ahrf.1608

ISSN : 1952-403X

Éditeur :

Armand Colin, Société des études robespierristes

\section{Édition imprimée}

Date de publication : 1 septembre 2005

Pagination : 25-51

ISSN : 0003-4436

\section{Référence électronique}

Frédéric Derne, «La chanson, " arme » révolutionnaire et chambre d'écho de la société en Auvergne », Annales historiques de la Révolution française [En ligne], 341 I juillet-septembre 2005, mis en ligne le 15 septembre 2008, consulté le 23 avril 2022. URL : http://journals.openedition.org/ahrf/1608 ; DOI :

https://doi.org/10.4000/ahrf.1608

Ce document a été généré automatiquement le 23 avril 2022.

Tous droits réservés 


\title{
La chanson, « arme » révolutionnaire et chambre d'écho de la société en Auvergne
}

\author{
Frédéric Derne
}

1 Mariage entre deux muses - Euterpe et Polymnie -, la chanson est considérée durant l'époque moderne comme l'art de ceux qui n'ont pas la parole, un moyen efficace de tempérer le gouvernement absolutiste. Quand la Révolution éclate, dans l'élan, l'enthousiasme et sous l'influence d'une nouvelle législation, l'esprit des mazarinades rebondit, avec une maturité politique accrue, dans les faubourgs, les ateliers, les sections révolutionnaires. Durant la décennie, la violence des sentiments populaires fait surgir des chants qui soutiennent les passions ardentes de l'époque, « la Révolution sonne l'heure de gloire de la chanson politique et sociale» ${ }^{2}$. Médium révolutionnaire par excellence, utilisant des airs à la mode, faciles à retenir, la chanson rend compte des événements, oppose révolutionnaires et contre-révolutionnaires au point de devenir un miroir de la société. Si Paris fait office de berceau de ces chansons, on chante aussi en Auvergne. En 1789, les chansons fredonnées au coin des rues et dans les théâtres, ou celles dont le public auvergnat s'enquiert dans la presse, sont truffées d'allusions politiques. Les différents modes de diffusion des productions imprimées, seules archives disponibles pour l'historien, témoignent de l'intense circulation de ce «journal de l'analphabète » dans la région. Les programmes des fêtes, les registres des sociétés populaires et, dans une moindre mesure, la presse régionale dans lesquels apparaissent les chansons viennent combler les lacunes d'un outil historique fondé sur l'oralité. Rapportées aux 2903 chansons recensées par Constant Pierre ${ }^{3}$, la production auvergnate durant la décennie s'élève à 158 pièces, et celle des œuvres proprement révolutionnaires ${ }^{4}$ à 112.

I - Diffuser pour mieux régner : inventaire, organes et principaux foyers de la chanson en Auvergne.

2 Les productions politiques et satiriques, en vogue dans la capitale, atteignent sans difficulté les contreforts du Massif Central ${ }^{5}$ sans que l'Auvergne, circonscrite au cadre 
administratif et séculier de la généralité de Riom, échappe à la frénésie musicale du moment - avec toutefois beaucoup moins de chanteurs de rues et de débitants de chansons que dans la capitale. Si les yeux (et accessoirement les oreilles) restent rivés sur la capitale, théâtre principal de la Révolution, les compositions n'oublient pas de relater des événements régionaux dignes de susciter l'émotion. La plus expansive de ces compositions est l'œuvre d'un sans-culotte - chantée à Clermont le 24 septembre 1793 au cours d'un repas donné par la section collège - louant l'influence de Couthon sur son département dans le règlement de la crise fédéraliste ${ }^{6}$ en présence de celui-ci. Malheureusement, la plupart des couplets d'inspiration locale n'ont pu bénéficier d'une transcription écrite. Les compositions imprimées restent le fait d'administrateurs, de versificateurs indigènes - parmi lesquels figurent en bonne place Nourry, Arnaud, Rabany, Clément... - qui n'ont de cesse de témoigner leur zèle pour la cause nouvelle. Si l'on occulte 16 œuvres tirées du Petit Chansonnier et deux romances (qui ne possèdent aucune valeur historique), 20 chants, hymnes, odes ou couplets indigènes, c'est un corpus de 112 pièces qu'il est possible d'isoler finalement.

3 Journaux ${ }^{7}$, recueils, programmes et procès-verbaux des grandes célébrations révolutionnaires et républicaines ${ }^{8}$, pamphlets... constituent autant de vecteurs de diffusion de cette production indigène (annexe 1 ). La capitale auvergnate n'est pas non plus épargnée par ce que la police a l'habitude de qualifier de troubles aux spectacles. Avec la Révolution, les passions théâtrales sont moins sociales que politiques, toujours vives, surveillées par les commissaires de police. En l'an IV, les royalistes investissent la comédie clermontoise pour en faire un de leurs lieux de rencontre. Les désordres redoublent en brumaire an V (octobre-novembre 1796); on interrompt les airs patriotiques, préférant Cadet Roussel et l'on couvre de lazzi ${ }^{9}$ le commissaire exécutif qui s'interpose: "Cadet Roussel a un cheval qu'est officier municipal». Il faut cependant savoir raison garder quant à la diffusion des chansons : aucune source n'est capable de mesurer la popularité ou le degré de diffusion des œuvres. S'il est vrai que le trajet d'hymnes envoyés par ballots entiers depuis Paris épouse les grands axes de communication, comment apprécier au-delà l'impact de la transmission par le «bouche à oreille», organe de vulgarisation privilégié des chansons? Les procèsverbaux des fêtes, souvent trop laconiques et peu fiables n'élucident guère le problème : lus le 2 pluviose an VII (22 janvier 1799), Rabany et Raymond le sont aussi à Aigueperse, Ardes, Arlanc, Augerolles, ou à Saint-Germain-l'Herm.

4 La comparaison entre compositions "parisiennes » et auvergnates s'arrête aux fluctuations des productions durant la décennie, celles-ci connaissant des destins paradoxalement similaires et croisés ${ }^{10}$ (annexe 2).

5 Quant à la censure, elle semble ne prendre qu'un caractère purement formel jusqu'au 9 Thermidor. Plus que le contrôle policier implicite omniprésent, c'est bien «l'efficacité» d'une autocensure dans les moments critiques qui prévaut: non imposée ni même suggérée par les gouvernements républicains, on note une certaine réticence ou impuissance chez les chansonniers à aborder des événements pénibles ou les décisions choquantes des autorités.

«La musique a toujours posé des questions gênantes aux sciences humaines car elle échappe à la rigueur conceptuelle» ${ }^{11}$. L'exploitation de cette source documentaire se heurte à deux difficultés principales ${ }^{12}$ : la première tient à la nature même de la production chansonnière jusqu'à l'invention de l'enregistrement sonore; le corpus est de surcroît difficilement accessible car les chansons connaissent un tirage limité sur 
des supports fragiles, et sont souvent disséminées sans classement spécifique dans les fonds d'archives. La grande majorité des chansons imprimées (ou manuscrites) de ladite période reste inséparablement liée aux programmes et procès-verbaux des fêtes versés dans la série $\mathrm{L}$ des archives départementales au chapitre «Fêtes publiques» pour le Puy-de-Dôme, ce qui n'est pas le cas dans le Cantal. On rencontre des chansons contre-révolutionnaires provenant de lettres interceptées ou d'hymnes tirés des principaux journaux régionaux de l'époque, des indications quant à la place accordée à la chanson dans les théâtres et les troubles qu'elle suscite, des chansons extraites du registre des sociétés populaires au hasard des dossiers de la même série. Des fonds spéciaux (privés) légués aux archives ou à la bibliothèque municipale et interuniversitaire de Clermont-Ferrand complètent ce maigre corpus en offrant quelques recueils de chansons ${ }^{13}$.

7 L'estimation de la valeur documentaire de ces œuvres musicales constitue la seconde embûche. Dans un contexte de piètre alphabétisation «se pose la question des relations troubles que la chanson entretient avec l'opinion publique: mode d'expression ou moyen de manipulation?» ${ }^{14}$. Il convient donc d'user avec circonspection et modestie de ces précieuses sources documentaires: les chansons politiques, toujours créées dans une situation concrète, ne se comprennent presque jamais isolément, mais seulement en relation avec le contexte ou avec des chansons qui les ont précédées. Le meurtre d'une ci-devant religieuse, mariée et enceinte, par son mari, en prairial an V (mai-juin 1797), ravive le mouvement anticlérical. Etienne Chabozi ne veut pas d'enfant; il épouse Anne Durif, de vingt ans son aînée, dans l'espoir d'un héritage. Les lois de succession anéantissent ses espoirs; il ne trouve d'autre moyen que d'éventrer sa femme à l'aide d'une fourche. Le journal républicain l'Ami des Lois donne une tout autre version pleine d'anticléricalisme à l'horrible assassinat ${ }^{15}$ afin de contrebalancer l'audience grandissante des réfractaires sur les communautés villageoises ${ }^{16}$.

8 Loin de tout déterminisme géographique, la carte des lieux de production des chansons auvergnates (annexe 3) confirme la prépondérance des villes situées à proximité des voies de communication, exception faite des quelques chefs-lieux de communes rurales (Tallende, Plauzat et Montel-de-Gelat soit 2,68\% de la production contre $60,7 \%$ aux cités d'au moins 5000 habitants). La faute en incombe à un réseau routier peu dense, mal entretenu à l'aide duquel il est impossible de contrôler le territoire ${ }^{17}$. Le niveau d'instruction ou au moins d'alphabétisation de la population, comme l'existence de structures d'accueil des idées philosophiques, la dichotomie entre une «élite culturelle» urbaine très minoritaire et la masse attachée à ses croyances et à ses habitudes participent tout autant à la suprématie des compositions citadines. Cette supériorité est autrement perceptible lorsqu'en novembre 1793 les représentants en mission dans le Puy-de-Dôme, Couthon et Maignet, se heurtent à un mur d'incompréhension un peu partout dans les campagnes lors même qu'ils organisent les fêtes décadaires dans lesquelles la chanson tient une place de premier ordre. Très rapidement, ils sont contraints de cantonner leurs actions aux abords immédiats de la ville. Il est peu étonnant dès lors que le chef-lieu du département du Puy-de-Dôme jouisse d'une supériorité culturelle incontestable et incontestée; les chansonniers clermontois composent plus du quart de la production totale de la région avec 39 hymnes loin devant Aurillac (13 réalisations) qui se dispute la prééminence en Haute-Auvergne avec Saint-Flour, Brioude et Pont-du-Château (rebaptisée Pont-sur-Allier). 
II- Des commissaires culturels : sociologie des auteurs1. Un chansonnier de ville attitré?

Alice Garrigoux, dans un article pour la Revue de Haute Auvergne ${ }^{18}$, énonce qu'un «historien des clubs a noté que chacun d'eux avait eu son poète». Ces chansonniers trouvent dans la composition une occasion d'afficher un républicanisme de tous les instants : versifier se révèle un instrument de promotion efficace pour les plus zélés de ces chansonniers-administrateurs.

10 Le chaleureux Lakairie ${ }^{19}$, assisté du musicien Crémont, orchestre de main de maître la fête de la Fédération de 1791 à Aurillac. Metteur en scène de «formation", il franchit le pas pour déclamer une ode, le 12 pluviôse an II (31 janvier 1794), en l'honneur du représentant en mission Bo lors d'une séance de la société populaire ${ }^{20}$. De tendance girondine, il semble dès lors tiraillé entre ses opinions et sa fonction officieuse de chansonnier attitré. Son modérantisme, son esprit vif et caustique ne tardent pas à le désigner à la vindicte des tenants du pouvoir qui le font emprisonner ${ }^{21}$. Revers de sa notoriété soudaine, quatre couplets chantés lors d'un banquet ${ }^{22}$ «de bons républicains sans culottes ennemis des traîtres et des fripons le 12 pluviôse an II» (soit le jour même de la venue de Bo à la société populaire) sont la cause de son incarcération. Remis définitivement en liberté par un arrêté du Comité de Sûreté générale du $2 \mathrm{e}$ sansculottide (19 septembre 1794) ${ }^{23}$, Lakairie peut à son aise louer le Directoire et célébrer le retour en grâce de ses compagnons politiques girondins autrefois proscrits dans des couplets chantés dans la séance du peuple de la commune d'Aurillac, le 1er frimaire an III (21 novembre 1794) de la République ${ }^{24}$. Il contribue dès lors à favoriser par la plume la période de réaction thermidorienne par l'entremise du Décadaire du Cantal, hebdomadaire fondé en ventôse an III (février-mars 1795) dans lequel il dispense quelques couplets de son inspiration.

11 Les parcours de Clément à Pont-sur-Allier ou de Talairat dans le Brivadois offrent des similitudes. Le talent littéraire et artistique de François Maximin Honoré Clément ${ }^{25}$ lui permet de nouer les liens de sociabilité nécessaires à son intégration communautaire. Seuls deux hymnes - datés de 1797 et 1798 - sur les huit de sa composition témoignent de son passage sur les rives de l'Allier. Son abnégation républicaine, dont la manifestation la plus évidente réside dans sa production musicale, contribue grandement à sa nomination au poste de secrétaire de l'administration municipale du canton en l'an V, puis à celui de commissaire du canton de Pont-sur-Allier en pluviose an VII (janvier-février 1799) - il en sera cependant remercié au bout de deux mois et sept jours -.

12 Catalyseur culturel régional, Clermont-Ferrand accueille pléthore de paroliers accoutumés à la pratique du vaudeville; grâce à Nourry, Verny, Arnaud, Boutarel puis Clément perdure le vaste et officieux chantier propagandiste impulsé par Paris ${ }^{26}$.

2. Les administrateurs locaux chantres de la Révolution

13 La propagande a ses raisons que l'art ignore ${ }^{27}$ : c'est en tenant compte de la fonction des hymnes révolutionnaires qu'il convient de les étudier et éventuellement de les juger ; «poète du peuple, ton premier devoir n'est-il pas d'être populaire et de sacrifier, s'il le faut, à ce but sacré quelques beautés poétiques d'un ordre trop élevé» s'interroge Grétry.

14 Là apparaît le point faible de la chanson révolutionnaire en Auvergne. Elle reste l'œuvre de ceux qui savent lire, écrire, composer, petits bourgeois ardemment républicains qui ignorent ce qu'est la faim. Aucun d'eux n'écrit en patois comme cela se 
produit en Basse-Corrèze avec la célèbre Marseillaise dite des "paysans». L'expression populaire sans-culotte se trouve réduite à la portion congrue ${ }^{28}:$ en dehors des ouvriers de la fabrique d'armes de Clermont, du sans-culotte Grou d'Aurillac ou d'Alligier, l'instituteur aux cent livres de patrimoine, peu louent le régime de l'an II. La figure de l'administrateur domine le tableau sociologique des paroliers : son lyrisme sert la cause directoriale à l'occasion des grandes célébrations républicaines. Temps forts du ralliement populaire, elles sollicitent le savoir-faire pédagogique des professeurs, instituteurs et membres de jury d'instruction.

15 Pierre Fontanier ${ }^{29}$ possède à ce titre un parcours atypique; prêtre de formation, la Révolution lui offre l'opportunité de participer à la vie publique. Né le 2 novembre 1765 à Moissac, son père occupe l'emploi de premier agent municipal à la Révolution. De par sa mère, il appartient à un milieu clérical. Il fait ses études au collège royal de SaintFlour où il est admis régent. Formé à l'humanisme, ce prêtre devient naturellement adepte des grandes idées du siècle qu'il propage à ses débuts dans l'enseignement en 1787. Principal fondateur de la société populaire de Saint-Flour, il devient secrétaire de l'évêché et vicaire épiscopal. En mars 1794, il se trouve à Paris où il a été proposé le 28 ventôse an II (18 mars 1794) par le comité d'instruction publique comme professeur d'histoire, mythologie et géographie à l'Institut provisoire du district de l'Égalité (anciennement collège Louis-le-Grand). À la fin de son stage à l'École normale, il est même nommé professeur de grammaire à l'École centrale de l'Ardèche.

Un hymne de sa composition rencontre quelques succès puisqu'il est chanté en 1797 à Clermont (peut-être pour la fête des époux) ${ }^{30}$. On sait par ailleurs que celui-ci est diffusé à l'occasion du mariage des prêtres à Aurillac et Mauriac. Les manifestations qui accompagnent le mariage de Fontanier avec la sœur Marie Artonne paraissent d'un tel intérêt qu'elles font l'objet d'un compte rendu imprimé par la société populaire de Saint-Flour. La veille de son mariage, Fontanier lui-même prend la parole dans la salle des séances de cette assemblée pour expliquer sa conduite et donne lecture du fameux hymne républicain ${ }^{31}$ :

«Je vais accomplir un des premiers devoirs de la nature humaine. Demain mes destinées seront unies à celles d'une compagne. Les républicains Montagnards de Saint Flour, qui se sont montrés constamment à la hauteur de la Révolution, ne verront pas avec indifférence un prêtre sensible et patriote s'attacher à la société par les nœuds les plus saints de la nature et du sang. Ce serait faire injure à leur civisme et à leurs lumières, que de m'attacher à combattre, auprès d'eux, le plus absurde et le plus barbare des préjugés, consacrés jusqu'ici par l'ignorance et le fanatisme [...] Je me flatte qu'ils voudront bien agréer l'hommage d'une hymne vraiment républicaine, que je leur offre, comme la nouvelle expression de mes sentiments et de mes principes».

3. Des impulsions bienvenues

17 Le souffle centralisateur parisien berce la production musicale locale. Objets d'éloges de la part de leurs concitoyens, les représentants en mission donnent le ton : ils organisent le calendrier des fêtes, composent quelques vers chantés lors de banquets civiques dressés en leur honneur ${ }^{32}$. Leur passage encourage les administrateurs auvergnats à rivaliser d'un zèle musical intéressé: la venue du thermidorien Musset à Aurillac suscite la composition par Lakairie de quelques vers chantés ${ }^{33}$.

18 L'attention et l'intérêt que portent les députés "parisiens" envoyés dans les départements aux réjouissances civiques ne sont rien en comparaison de l'impulsion donnée par le ministre de l'Intérieur François de Neufchâteau - et relayée par les 
administrations centrales -. Ses circulaires sont des modèles de précision par l'exposé du mobile des fêtes, du projet que se proposent d'atteindre les administrateurs. Le nouvel élan donné à la composition ne trouve qu'un faible écho populaire ; la verve des tenants locaux du pouvoir directorial se heurte trop rapidement à un mur d'incompréhension tant l'art impulsé devient un art dirigé.

Pâles imitations des grands hymnes nationaux, les couplets sont le fait d'hommes à la solde du régime qui se soucient davantage de leur carrière que des idées qu'ils prétendent défendre. La versatilité se manifeste d'ailleurs clairement chez l'un d'eux qui vire de bord au gré des aléas politiques. Jean-François Talairat ${ }^{34}$, fervent défenseur des idéaux révolutionnaires, devient par la suite adjoint (1807 à 1813) puis maire de Brioude (1813 à 1823); il est anobli par Louis XVIII en 1818, son titre de baron enregistré en 1830. Grand bourgeois, révolutionnaire, républicain, bonapartiste, royaliste, il n'oublie pas d'être poète et chansonnier. La chanson peut-elle acquérir un certain degré de persuasion lorsqu'elle se trouve stéréotypée, incapable de soulever l'émotion des masses? Que faire lorsque les administrateurs républicains eux-mêmes doutent de la véracité de leur propos et préfèrent écrire par opportunisme?

III- L'Auvergne au diapason1. De l'importance du timbre

Se gardant de donner une musique nouvelle - de surcroît auvergnate - à leurs œuvres, les chansonniers usent des timbres d'opérettes célèbres ou d'autres airs chéris de la Révolution comme support musical. Le choix du timbre, du moins son titre, est fondamental puisqu'il tend à renforcer la pensée dominante des paroles. Les Jacobins louent en 1794 leur faction au son de Fiers habitants de la Montagne ${ }^{35}$. Réactionnaires et royalistes trouvent dans le Juif errant ${ }^{36}$ une arme en vue de vilipender le comportement des prêtres constitutionnels tandis que le timbre Je l'ai planté, je l'ai vu naître, empreint de nostalgie ${ }^{37}$, soutient la thèse de la fondation séculaire de la royauté .

21 Le doute persiste concernant quelques timbres tels Le cour de Nanette ${ }^{38}$, Une petite fillette ${ }^{39}$, Je t'aime tant ${ }^{40}$ ou Actamini ${ }^{41}$, mais il ne semble pas y avoir eu de création proprement auvergnate. Les airs chéris des républicains font l'objet d'abondantes parodies (respectivement 19 et 9 reprises de La Marseillaise ${ }^{42}$ et du Chant du Départ ${ }^{43}$. C'est du répertoire des théâtres lyriques que les chantres de la Révolution tirent une bonne part de leurs emprunts, la plupart postérieurs à 1780 comme Avec les jeux dans le village ${ }^{44}$ de Piis, l'Air des dettes de Champein (1787), celui de La croisée de l'avocat Ducray-Duminil. D'autres airs proviennent de pièces de théâtre contemporaines comme Comment goûter quelques repos, Vous qui d'amoureuse aventure provenant de Renaud d'Ast (1787) ou de l'opéra comique: Richard cour de Lion (1784), de Grétry (son célèbre $\hat{O}$ Richard [ $\left.{ }^{45}\right]$ en témoigne). Les chansonniers auvergnats se réapproprient également des airs de chansons plus anciennes : L'Hymne des Versaillais ${ }^{46}$, de Girout, commençant par les vers «Quels accents, quels transports !» reste le plus prisé des auteurs régionaux.

Si les chansonniers de la Révolution empruntent nombre de timbres à leurs devanciers, ils en fournissent certains à leurs contemporains auvergnats : Eh! quoi tu peux dormir encore $^{47}$, Français laisseras-tu flétriir ${ }^{48}$, Français le signal est donné ${ }^{49} . .$.

L'innovation musicale, sans être bannie, cède le pas aux missions sociales et politiques de la musique à l'occasion de grandes liturgies civiques.

2. Un moyen d'éducation : l'hymne durant la fête

24 Spectacle total où les spectateurs sont en même temps acteurs, la fête révolutionnaire se veut participative. Pour produire cette «dilatation des esprits», cette «vive agitation», cet « éveil éclatant» qui signalent une fête réussie, il faut agir sur tous les 
sens à la fois, multiplier les impressions et les émotions ${ }^{50}$. Fête et musique entretiennent des liens étroits en Auvergne, les grands rassemblements révolutionnaires offrant à Orphée une place de première importance. Les programmes gagnent en solennité ce qu'ils perdent en spontanéité ; projets et relations de fête sont comme des tableaux qu'on ne pourrait voir ou des musiques qu'on ne pourrait entendre mais dont on n'aurait qu'un descriptif, minutieux certes mais toujours un peu froid. Une chose est sûre, les programmes règlent les fêtes comme du papier à musique! Certains procès-verbaux laconiques imaginent plus qu'ils ne décrivent la cérémonie à laquelle ils se réfèrent ${ }^{51}$.

$\mathrm{Au}$ service de l'administration, tour à tour complément du discours ou simple ornement donnant un caractère émotionnel à des cérémonies officielles emphatiques, l'hymne tend à se substituer à la chanson pour devenir le principal instrument musical révolutionnaire dans la région. Mais en donnant en spectacle la Révolution, le gouvernement signe l'arrêt de mort de la chanson populaire dans sa composition ou son acceptation par le peuple. Les paroliers auvergnats ont beau user des airs chéris de la Révolution ${ }^{52}$, réception et expression populaires deviennent de véritables arlésiennes de la chanson ${ }^{53}$; «presque toujours cela sonne creux, malgré les allures pompeuses de la versification» ${ }^{54}$. Le rêve d'une musique à la fois art pédagogique et de propagande s'estompe lorsque les compositions s'enferment dans une versification stéréotypée qui fait le jeu - à l'image des fêtes - de la tradition...

Les chansonniers emploient images allégoriques et autres métaphores, mais l'utilisation du manichéisme entre un Ancien Régime honni et une expérience républicaine annonçant des jours meilleurs semble le plus à même de vulgariser des idées simples. Opposition entre la servilité d'Ancien Régime et la naissance de l'homme nouveau, passage d'un régime tyrannique à celui des arts et des bonnes mœurs, antagonisme entre une société féodale d'oppresseurs et une République vertueuse, légitiment tour à tour le nouveau gouvernement (annexe 5).

3. Le journal chanté de la Révolution en Auvergne : combats, aspirations et résistances Vecteur de la culture populaire, la chanson donne au plus grand nombre le moyen d'entrer dans le débat politique et d'exprimer craintes, espoirs, acceptation ou rejet d'une politique... «Le seul répertoire musical de la Révolution française qui soit réellement la chambre d'écho de l'histoire et qui reste à la portée du plus humble citoyen, c'est la chanson» ${ }^{55}$.

28 À l'heure où celle-ci s'en empare, la lutte est déjà engagée entre partisans des prêtres réfractaires et jureurs. Les premiers, blessés dans leurs convictions, témoignent par des couplets ironiques et satiriques le mépris que leur inspirent les ecclésiastiques qui croient pouvoir prêter le serment exigé par l'Assemblée nationale. Ces chansons, composées peut-être dans les maisons de réclusion, circulent en copies manuscrites et se déclament lors de réunions intimes. Sans briller par leur inventivité, leur style ou la versification, ces productions intéressent l'historien en ce qu'elles permettent de mesurer l'accueil réservé aux empiètements de l'Assemblée constituante sur le terrain de la religion par une partie des citoyens auvergnats.

Ces querelles chansonnières pour le gain d'une légitimité cultuelle font rage avec une grande acuité dans le Cantal. Des couplets publiés dans le journal le Cantaliste de PagèsVixouze ${ }^{56}$ répondent à un pamphlet datant de $1791{ }^{57}$ portant atteinte à l'intégrité de l'évêque constitutionnel Thibault. 
«Allons tous à la chasse

De l'enragé Thibault

Le voilà qui nous passe

Il franchit tout d'un saut

C'est un chef de la meute

De défunt Mirabeau

Que déjà l'on ameute

Contre notre Ruffo. »

Le pamphlet dépeint Thibault comme une bête qu'il s'agit de traquer, un sbire de Mirabeau. Mort le 2 avril de la même année, Honoré Gabriel Riquetti comte de Mirabeau incarne encore pour l'auteur cet homme qui réplique à Dreux-Brézé dans la salle du Jeu de paume - et non le traître auquel les adversaires reprochent de toucher de l'argent de la cour à la veille de sa disparition-, le promoteur de la Révolution de 1789 et de ses réformes au premier rang desquelles figurent l'abolition des droits féodaux, la création des assignats et la confiscation des biens de l'Église; le pamphlet accuse Thibault d'épouser la vie toujours plus dispendieuse et plus dissolue du tribun. Le caractère bestial discrédite un clergé constitutionnel émissaire de la Législative se livrant à la curée d'un prélat qui recueille les suffrages populaires. Il incarne un animal dangereux qui disperse son troupeau de fidèles. La caricature politique s'impose comme une arme politique dans ce grand combat de la satire que chaque camp engage aux dépens de son ennemi ${ }^{58}$. Le jureur est accablé de défauts antagoniques au comportement attendu du religieux. Schismatique, il devient un intrus, un agent des Jacobins. La possibilité pour le prêtre de rompre son vœu de chasteté constitue un autre leitmotiv du discours réfractaire ${ }^{59}$.

Trois années durant, les résistances à la mise en œuvre de la Constitution civile du clergé, les appels à la désobéissance voire à la guerre civile travestie en guerre sainte lancés par les "fanatiques", alimentent les chroniques des troubles de l'ordre public. L'Assemblée législative, bientôt relayée par la Convention, mène une politique de coercition à l'égard des réfractaires à partir d'août 1792, synonyme d'émigrations, réclusions, incarcérations, déportations. Dans les régions, les représentants jouent les chefs d'orchestre que la propagande des paroliers répercute. Maignet et Couthon quittent la Ville Affranchie le 3 novembre 1793 pour retourner dans le Puy-de-Dôme afin d'y procéder à une cure révolutionnaire selon l'expression de ce dernierrenouvellement des autorités constituées et surtout défanatisation du départementqu'ils entament par une première étape à Ambert le 15 brumaire ( 5 novembre). «Dans un climat d'euphorie, Couthon fut porté, comme un triomphateur, dans les bras de fervents sans-culottes» ${ }^{60}$ et publie un étrange pamphlet anticlérical interprété sur l'air de l'hymne de la Liberté. Les tribuns décident, le 18 brumaire an II (8 novembre 1793), l'impression et la diffusion en quatre mille exemplaires de cette pochade intitulée les Litanies des Saints convertis en monnaie destinée à être envoyée à toutes les communes du département. Le véritable auteur des couplets passe pour être le député de la HauteLoire Balthazar Faure ${ }^{61}$ (annexe 6). La prééminence du «décret» qui s'abat sur les saints tel une guillotine ne laisse que peu d'alternatives au catholicisme; il faut se soumettre à la Convention et à ses membres. Les saints les plus célèbres n'échappent pas à cet autodafé. La France en guerre souffre d'un besoin de numéraire que peut pallier l'alliage de «l'antimoine» - jeu de mot volontaire - et des objets précieux du culte mis à disposition de la Nation. La violence des termes employés révèle l'ampleur de la 
flambée déchristianisatrice qui s'empare de la région sous l'impulsion des représentants en mission; tout est fait pour tourner en dérision la superstition ${ }^{62}$. La chanson illustre la radicalisation de la politique anti-religieuse de Couthon à la fin de l'année 1793. Lors du compte rendu qu'il dresse de son action devant la Convention, il juge avoir remporté, avec les armes de la raison et celle d'une croyance sur la nature des choses, une victoire totale sur le fanatisme et la religion. "La tonalité irrévérencieuse et voltairienne de cette intervention à l'Assemblée [ainsi que la diffusion de la chanson] montre l'état d'esprit de Couthon fin $1793{ }^{63}$.

Les paroliers auvergnats s'emploient à mobiliser la population locale pour une guerre lointaine qui ne menace plus guère les frontières à partir de 1794. Il faut attendre les années directoriales - à l'exception du couplet de Delolm Labaudie sur la prise de Toulon en $1793{ }^{64}$ - pour que les thèmes de la traîtrise ennemie, d'une menace imperceptible, deviennent récurrents dans les compositions ${ }^{65}$. "On pense concrètement à l'Europe militaire coalisée contre la République. L'idée de complot de l'étranger est pour Barère constitutive de la Révolution, guerre idéologique de la République contre le royalisme» ${ }^{66}$. La chanson, loin d'adoucir les mœurs incite à la vengeance, la punition et l'anéantissement de l'ennemi. La France doit sacrifier ses enfants pour perpétuer la République et éloigner la menace royaliste. Les Français n'ont pas le choix, il faut vaincre ou mourir. À l'image d'une liturgie civique qui glorifie les martyrs de la liberté, les chansonniers auvergnats vont célébrer les héros qui portent les idéaux de liberté et d'égalité hors des frontières.

Les couplets sur le sujet n'ont de cesse de placer la France dans une attitude défensive. Le soldat se contente de rendre coup pour coup à l'adversaire, ce qui légitime les sentiments de vengeance que nourrissent les paroliers auvergnats: «le passage de l'armée d'Italie par Clermont et l'assassinat des plénipotentiaires français à Rastatt ( 9 floréal an VII - 29 avril 1799) fournirent aux administrateurs départementaux les éléments indispensables à leur propagande, autant nationaliste que patriotique: glorifier l'armée victorieuse, venger les Français des traîtres autrichiens justifiaient, dans l'optique des militaires et du pouvoir civil, l'engagement des citoyens» ${ }^{67}$. L'assassinat des ministres offre une occasion inespérée de renforcer la perfidie, la cruauté d'un ennemi bafouant les règles diplomatiques ${ }^{68}$. Les étrangers sont présentés comme des créatures sans foi ni loi à éliminer. La France a le devoir de laver l'affront. Saint-Just a depuis longtemps élaboré un réquisitoire en établissant une adéquation parfaite entre les termes étrangers et tyrannie; désigner l'étranger, ce n'est plus simplement désigner des ennemis extérieurs, ceux qui se désolidarisent de la communauté révolutionnaire et s'isolent, mais c'est encore désigner tout le champ des valeurs morales, sociales et politiques qui s'opposent à l'établissement de la République. Dans les chansons, l'étranger symbolise à la fois l'idéologie contrerévolutionnaire et les acteurs qui portent ce contre-projet ou qui simplement font obstacle au projet révolutionnaire.

La vengeance contre l'ennemi est-elle le meilleur moyen d'encourager la conscription et de redonner un peu d'enthousiasme à la population dans le but de soutenir l'effort de guerre ? Assurément non si l'on se fie aux mutilations, aux désertions des soldats de la Révolution en Auvergne. Les municipalités mettent beaucoup de mauvaise grâce à dresser la liste des conscrits, les établissent souvent avec négligence ${ }^{69}$. La patrie n'est plus en danger même si elle doit combattre une armée de rois coalisés. La mobilisation 
des Auvergnats par la chanson sonne d'autant plus faux que les hymnes républicains rendent parallèlement gloire à l'armée victorieuse.

La Révolution et l'Empire, en transformant de fond en comble la manière d'être des armées et en établissant, par la conscription, un nouveau mode de recrutement, contribuent à un renouvellement radical des chansons militaires avec la naissance de la chanson de conscrit. La verve d'Armand ${ }^{70}$, sur l'air de Méhul, accompagne le départ des bataillons du Puy-de-Dôme pour le front en décembre 1799. "Ce qui caractérise l'homme libre, c'est son aptitude à exercer le "métier de citoyen" dans sa double configuration civile et militaire ${ }^{71}$; après le guerrier qui expose sa vie pour défendre les moissons, nul mérite mieux de la patrie que celui qui les fait naître. La parabole du citoyen-soldat romain introduit les épineuses questions de la conscription et des subsistances - essentiellement lors de la fête de l'Agriculture - au cœur du discours chansonnier. L'agriculteur, nouveau Cincinnatus prêt à répondre à l'appel de la conscription si le besoin s'en fait sentir, tire de son labeur les vivres nécessaires pour soutenir l'effort de guerre. Vulgarisées à l'occasion des fêtes décadaires, ces références récurrentes trouvent un bien faible écho dans l'auditoire ${ }^{72}$. Ce discours ne peut obtenir l'assentiment du menu peuple auvergnat tant il est étranger à ses préoccupations quotidiennes - dès l'automne 1792, les problèmes de subsistance s'accumulent dans la région -.

Bon nombre de chansons satiriques sont diffusées sous couvert de l'anonymat par des contre-révolutionnaires. S'il s'avère aisé d'arrêter un chansonnier professionnel royaliste - Ange Pitou laisse ainsi son bonnet de nuit à la prison du Temple -, les tenants du pouvoir font preuve d'impuissance face à des feuilles volantes anonymes ou à des phrases chantées au coin des rues ${ }^{73}$; au mieux, les autorités retrouvent-elles le destinataire de la lettre, mais comment punir le véritable auteur? Ces couplets en vogue durant la Terreur rencontrent un succès certain dans le Cantal par la réprobation qu'ils expriment à l'encontre du gouvernement révolutionnaire ${ }^{74}$.

« Raison à quoi sert ton flambeau

Qui doit, dit-on, jusqu'au tombeau

Éclairer l'homme sage

Dans son enfance à peine il luit (bis)

Dans la jeunesse il éblouit

Et s'éteint avec l'âge.»

L'auteur prend le contre-pied de la raison que prônent les révolutionnaires contre l'obscurantisme monarchique. «Dit-on» apparaît n'être qu'une rumeur, et non un fait avéré. Le texte souligne la dichotomie entre un discours montagnard utopique et le modèle de gouvernement qu'il propose. Les espérances placées dans les législateurs s'amenuisent au fur et à mesure que s'égrènent les années révolutionnaires, les lumières de la raison s'estompent dans les souffrances de la Terreur.

Symboles de la Nation assemblée, les airs chéris de la Révolution font également l'objet de parodies réactionnaires. Aucune occurrence n'atteste de la réutilisation de ces hymnes par les contre-révolutionnaires; mais les Thermidoriens profitent du 9 Thermidor pour «emprunter» les timbres de la Marseillaise et de l'hymne de Chénier et Méhul pour les employer à leur avantage. Il s'agit pour eux de s'élever contre les pratiques terroristes des Jacobins tout en adoptant une ligne de conduite républicaine digne du plus grand patriotisme. Les accents du Chant de guerre pour l'armée du Rhin 
rythment ainsi les couplets intitulés Le cri de mort contre les conspirateurs et les hommes de sang ${ }^{75}$. Auvergne durant la décennie. Les chansons politiques et satiriques, sans doute dans la continuité des années 1780 , héritent de la légèreté de l'opérette, de l'opéra comique en témoignent les timbres utilisés provenant pour la grande majorité de ce genre théâtral -. 1794 constitue une césure avec la reprise en main de la politique culturelle par le gouvernement; dès lors, l'hymne des fêtes décadaires ou nationales devient le support musical privilégié des auteurs auvergnats. Le style de ces œuvres s'éloigne de la conception populaire de la chanson. Se dégage alors une tendance, sous le Directoire, qui voit la chanson militaire prendre irrémédiablement le pas sur ses concurrentes. L'hommage aux défenseurs de la patrie, l'obsession de victoires et de guerres font du chant l'un des principaux instruments de louanges et de propagande des grands généraux de la Révolution. guerre européenne qui s'éternise et les problèmes de subsistances qu'elle génère. Le peuple ne pense qu'à la paix, à la stabilité et au bonheur. Une voie «impériale» semble offerte à un de ces grands héros militaires. En l'an VI, les campagnes auvergnates vouent un culte à ce général corse qui fait parler de lui sur le front italien. On suit ses pas d'Italie en Égypte. Il doit d'ailleurs ses premiers lauriers populaires à la signature de la paix de Campoformio (1797), paix que loue Lakairie dans ses couplets chantés le 12 brumaire an VI (2 novembre 1797) au théâtre de la rue de Lacoste à Aurillac ${ }^{76}$.

«Air : Dans le cœur d'une cruelle

O toi guerrier magnanime

Sage, humain Buonaparté

O toi dont le nom sublime

Vole à l'immortalité !

Reçois l'hommage

Du peuple triomphateur!

Oui sa gloire et son bonheur

Et son bonheur

Sont ton ouvrage. »

La chanson peut par l'émotion indifféremment détruire ou construire les États, pervertir ou édifier les hommes selon l'usage opportun qu'en fait le législateur ${ }^{77}$; elle apparaît comme un art de divertissement dont la permanence est remarquable : aucun autre n'est mieux accordé au caractère du peuple. La chanson est une arme, et devient pour lui un moyen d'affirmer collectivement des convictions. Elle participe à la critique, à la dénonciation des institutions en place, et joue, même inconsciemment, un rôle subversif et révolutionnaire. 


\section{ANNEXES}

Annexe 1

Imprimeurs, diffuseurs et collecteurs des chansons révolutionnaires auvergnates

Voir document annexe

Annexe 2

Fréquence décennale des compositions « parisiennes » et auvergnates

Voir document annexe

Annexe 3

Lieux de production des chansons en Auvergne (circonscrite à l'ancienne généralité de Riom)

Voir document annexe

Annexe 4

Tableau sociologique des principaux auteurs auvergnats en Révolution

Voir document annexe

Annexe 5

Tableau des thèmes récurrents présents dans les chansons auvergnates révolutionnaires (112 compositions étudiées)

Voir document annexe

Annexe 6

Litanies de Saints convertis en monnaie

Air : de l'Hymne de la Liberté

«Pierre, Paul, Mathieu, Mathias, Jude, Simon et vous Barthélemi, Voyez à quelle épreuve rude Les Français vous mettent aujourd'hui, En se moquant de St Rémi,

St Philippe, et vous frères Jacques, Jean de Jésus le bien aimé, Gros Thomas et vous cher André, Sts devant comme d'après Pâques.

Vos cris sont superflus, Vous serez tous fondus, Grands saints, (bis)

Dans le creuset

Tombez, c'est le décret.

St Marcel, Ste. Geneviève,

Sts Renommés de tous pays, 
St Roch et son chien son élève,

St Jean Baptiste et St. Denis,

St Jean de Latran et St. Prist,

Et vous cochon de St Antoine,

Ah! plus vous serez gros et gras,

Plus vous produirez de ducats,

Dans la fonte de l'antimoine.

Vos cris sont superflus,

Vous serez tous fondus;

Grands saints, (bis)

Dans le creuset

Tombez, c'est le décret.

Marthe, Marie, Magdelaine,

Femmes qu'adorait le Sauveur,

St Hubert, et vous Ste Hélène,

St Charlemagne, l'Empereur,

St Louis (nom qui fait horreur)

St Luc, St Gile et St Spire,

Papes, évêques et docteurs,

Consolez-vous de vos malheurs,

Curtius va vous faire en cire.

Vos cris...

Nous ne brûlerons plus de cierges

Devant l'autel de nos patrons,

Mais quand nous trouverons des vierges,

Oh, comme nous les chérirons!

oh, comme nous les fêterons!

Nous n'aimerons que les vivantes;

Les vivantes nous aimerons,

Et nos neveux qui surviendront,

Se les choisiront pour amantes.

Vos cris...»

\section{NOTES}

1.Annie LAMADON, « La fête en Révolution », Revue d'Auvergne, tome 103, numéro 1, 1989 , p. 59.

2.Michel Vovelle (dir.), L'état de la France pendant la Révolution, Paris, 1989, p. 159.

3. Constant Pierre, Les hymnes des fêtes et cérémonies de la Révolution française, Paris, Imprimerie nationale, 1908.

4.Nous entendons par révolutionnaires les chansons dont les couplets évoquent la situation politique de la France et de la région durant la décennie 1789-1799.

5.Le souffle centralisateur met en moyenne trois jours pour atteindre Clermont ; les députés jacobins ne se privent pas pour envoyer par ballots entiers à leurs concitoyens auvergnats les airs chéris de la Révolution quand ils n'utilisent pas les imprimeurs locaux - pensons à Limet proche de Couthon - comme vecteur de diffusion. Le réseau 
traditionnel du colportage, difficilement décelable dans les archives, a lui aussi joué son rôle. La Ronde pour la plantation de l'arbre de la liberté de Grétry est, par exemple, chantée dès 1793 dans la région tout comme la lamentation royaliste ô mon peuple qui circule sous le manteau.

6.BMIU, Fonds Paul Leblanc A31196, Sans-culotte, Veillons au salut de l'empire, septembre 1793, Clermont-Ferrand.

7. S Sauf une unique publication satirique, due à Abraham (Le Père Duchêne en patois) en l'an VII, le Puy-de-Dôme ne fait guère marcher ses presses à imprimer ", rappelle Philippe Bourdin et la chanson diffusée par la presse est proportionnellement faible. Nous avons rencontré cinq chansons reproduites dans les journaux, dont trois pour le seul décadaire du Cantal; il faut dire qu'avec un rédacteur tel que Lakairie, il ne peut en être autrement. Une chanson de Desfeorges nous est également connue grâce au Rédacteur, journal non régional.

8.Au premier rang desquelles figurent la commémoration de la fondation de la République, la célébration du 10 Août, la fête de la Fédération, celle de l'Être suprême... d'autres comme la fête des époux, de l'agriculture, de la jeunesse ou la mort de généraux tels Joubert, Hoche...

9.Philippe Bourdin, Des lieux, des mots, les révolutionnaires, Clermont-Ferrand, Institut du Massif Central, CHEC, 1990, p. 53.

10.Les travaux de Constant Pierre rendent compte de la progression régulière du corpus chansonnier parisien entre 1789 et 1793 jusqu'à son apogée en 1794. La production auvergnate est également portée à son faîte en 1794. La chute du Comité de Salut public n'y est pas étrangère. Les 29 chansons de L'heureuse Révolution du 9 thermidor, livret regroupant des œuvres essentiellement brivadoises sur l'événement, témoignent du renouveau de l'expression populaire en Haute-Loire ; ajoutées aux compositions des années 1793-94, elles représentent plus de la moitié des compositions régionales de la décennie (57 œuvres). Les années 1795-1796 constituent à Paris comme en Auvergne des temps de reflux pour la chanson. Au second apogée de la chanson auvergnate (30 compositions) correspond le sursaut patriotique parisien de 1799, date de la reprise en main de la politique culturelle par le ministre François de Neufchâteau. 11.Michelle BIGET, « la Révolution et ses musiques », La Pensée, numéro 253, septoctobre 1986, introduction.

12.Jean-Louis JAM, in Images de Robespierre, actes du colloque de Naples (27-29 septembre 1993) réunis par Jean EHRARD, Clermont-Ferrand, CRRR, p. 299.

13.C'est ainsi que le fonds Auvergne de la BMIU regroupe une bonne partie de la production chansonnière clermontoise ou des recueils comme l'Almanach des Muses et le Petit Chansonnier. Le fonds ancien possède quelques productions d'artistes pour la plupart parisiens dont les compositions ont été diffusées jusqu'à Clermont. Paul Leblanc a, quant à lui, récolté une trentaine de chansons essentiellement brivadoises concernant $L$ 'heureuse révolution du 9 Thermidor alors que les fonds Delmas et Leymarie offrent bon nombre d'entrées à ceux qui souhaitent percevoir la vie révolutionnaire cantalienne au travers de la chanson. La série L des archives départementales du Puyde-Dôme livre des réponses quant à l'aspect sociologique de la question. Les paroliers, pour la plupart administrateurs ou professeurs, figurent sur les registres au chapitre personnel, élections, instruction publique ou contributions. Les archives communales de Riom et Clermont-Ferrand affinent le profil-type de ces artistes d'un jour par l'étude des districts et des sections. Le fonds Paul Leblanc donne quant à lui quelques 
précieuses informations sur les bardes républicains de Brioude, alors que les fonds Delmas et Leymarie en font autant pour la Haute-Auvergne.

14.Jean-Louis Jam, op. cit., note (142).

15.Philippe Bourdin, Le Puy-de-Dôme sous le Directoire, vie politique et esprit public, Clermont-Ferrand, Mémoire de l'Académie des sciences, Belles-lettres et Arts de Clermont-Ferrand, 1990, p. 231.

16.«La version des faits donnée par L'Ami des lois, journal républicain à gros tirage, dans son numéro 682, si elle n'atténue en rien l'atrocité du crime, en impute la cause première au fanatisme religieux. L'idée d'une responsabilité ecclésiastique avait depuis longtemps fait son chemin, renforcée par des chansons de circonstance» (Marcel David, Fraternité et Révolution française, Paris, Aubier, 1987, pp. 126-127).

17.Philippe Bourdin recense deux routes de première classe (Paris-Le Puy et LyonLimoges) et deux de seconde classe (Clermont-Ambert et Clermont-Aurillac) en tout et pour tout. À l'inverse, les marges forestières notamment abritent réfractaires, émigrés, déserteurs et brigands.

18.Alice GARRIGOUX, «Une ville en Révolution : les jacobins d'Aurillac (1790-1795) », Revue de la Haute Auvergne, tome 52, Aurillac, janvier-septembre 1989, pp. 37-85.

19.Jean Baptiste Lakairie (1765-1822) est le fruit de l'union de Géraud Lakairie, choriste de l'église paroissiale, et de Marie Noël. Jean Delmas ne s'y trompe pas : « Grâce à la position qu'occupait son père il reçut une éducation libérale qui jointe à un certain esprit naturel ne tardèrent pas à faire de lui un fin lettré et un amant dévoué de notre histoire locale ».

20.AD Cantal, Fonds Leymarie 6J77, Lakairie, L'autre jour Lucas dans la prairie, janvier 1794, Aurillac.

21. «Je restais constamment attaché aux principes de liberté et de philosophie qui m'ont toujours animé. Mais comme je conservais toujours un caractère franc et indépendant je ne fus pas au grè des meneurs de 1793. Je fus donc épuré de la société notamment par le représentant Bo; je fus mis en réclusion et enfin rappelé à cette époque où je fus employé pour les fêtes» (ibid., Fonds Delmas 27J32).

22.Ibid., Fonds Delmas 27J31 (bis), Lakairie, Couplets chantés dans un banquet de bons républicains, Je ne forme point de désir, février 1794, Aurillac.

23.Il figure pourtant sur la liste des gens suspects à faire arrêter que l'on trouve chez Carrier lors de la saisie de ses papiers ; au numéro 18 s'étale en toutes lettres le nom de Lakairie avec cette mention indicative propre à faire envoyer à l'échafaud celui qu'elle désigne : émissaire dispendié par Roland pour distribuer des diatribes hypocrites, le prônant partout jusque dans les campagnes ; ennemi et calomnieur de la montagne ; grand artisan du marais : provocateur de la force départementale et du fédéralisme (ibid., Fonds Delmas 27J32).

24.Ibid., EDEP1500 (419), Lakairie, Français, le signal est donné, novembre 1794, Aurillac. 25.Né à Aix puis marchand « clincailler » à Marseille avant la Révolution, instituteur à Bousique (département de l'Hérault), on ne sait quelle fortune l'amène à Pont-surAllier en l'an IV. Présentant des qualités d'artiste mécanicien et de sténographe, il se propose de devenir instituteur de l'école primaire de sa commune d'adoption. Son implication dans la vie communale de Pont-sur-Allier lui ouvre les portes de l'administration départementale ; il suit Rouillon, ex-président de l'administration, nommé commissaire du département après le 30 prairial an VII (18 juin 1799). «Cette nomination sera la bienvenue, puisque dans le programme de la fête du premier vendémiaire an VIII figurent trois odes » de sa composition (Philippe BOURDIN, Le Puy- 
de-Dôme sous le Directoire, vie politique et esprit public, Clermont-Ferrand, Mémoire de l'Académie des sciences, Belles-lettres et Arts de Clermont-Ferrand, 1990, p. 152). 26.43\% des hymnes clermontois soit 39 compositions sont le fruit de leur verve. 27. Les fêtes de la Révolution, actes du colloque de Clermont-Ferrand (1974), réunis par Jean Ehrard et Paul Viallaneix, Paris, Société des études robespierristes, 1977, p. 437. 28.Les sept compositions de sans-culottes recensées coïncident avec l'avènement à l'échelle régionale des Montagnards entre juillet 1793 et juin 1794.

29.Bernard Vinatier, "Un intellectuel dans la Révolution du Cantal : Pierre Fontanier », Revue de Haute Auvergne, tome 52, janvier-septembre 1989, pp. 181-240. Dans une note à Jean Delmas, l'abbé Chabau mentionne parmi les déprêtisés : «Fontanier, de SaintFlour, prêtre marié qui envoie le 26 brumaire an II à la Convention un hymne de sa composition sur l'air des marseillais. Il paraît que cette pièce a été insérée au bulletin de la Convention mais je n'ai pu la trouver.»

30.BMIU, Fonds Auvergne A34934 (2), Fontanier, Le célibat Réprouvé, Marseillaise, 1797, Clermont-Ferrand.

31.AD Cantal, 205F1.

32.On trouve trace de sa présence, selon Martine Braconnier (Couthon de l'Auvergne à la Convention, Georges ou les métamorphoses de la raison, Yssingeaux, Roure, 1996), lors de la célébration de six fêtes, dont cinq à Paris. Des fêtes auxquelles il assiste et qu'il relate, parfois des fêtes qu'il propose ou qu'il impulse. L'attachement de Couthon à la commémoration du 10 Août est tel qu'il félicite les autorités de Clermont d'avoir organisé une fête digne de l'événement ; la veille de la commémoration, Limet, célèbre imprimeur de Clermont se trouve à Paris. Nul doute que les commissaires du Puy-deDôme, ainsi que Limet, réunis autour de Couthon, servent de liens entre Paris et Clermont, entre Couthon et la municipalité clermontoise à propos des festivités. Ce n'est pas un hasard si au cours de sa marche à travers la ville, le cortège s'arrête au milieu de la rue Ballainvilliers où se trouve l'appartement de Couthon et y entonne un hymne à la liberté. Cette rue reçoit d'ailleurs le nom de rue de la Liberté.

33.AD Cantal EDEP1500 (419), Lakairie, Français le signal est donné, novembre 94, Aurillac..

34.Jean-François Talairat (1766-1850), avocat dont le père a été subdélégué de l'intendant d'Auvergne, seconde activement Reynaud, représentant en mission montagnard en Haute-Loire, au moins par ses discours enflammés ; guère inquiété après le 9 Thermidor, il obtient le poste de commissaire du Directoire exécutif de Brioude en 1795 puis, trois ans plus tard, celui de président du Tribunal criminel de la Haute-Loire avant de devenir en 1800 juge suppléant au Tribunal civil. Révolutionnaire et républicain, il est en outre un homme de lettres. Dans ce domaine, il faut surtout retenir la véhémence de ses discours, très prisés des Brivadois. Discours parlés... mais aussi discours chantés. Il prend ainsi la plume le jour de la fête de l'Être suprême de Brioude pour composer trois hymnes (BMIU Fonds Auvergne A34934 (11), Talairat, Romance d'Estelle, Je suis Lindor, Avec les jeux dans le village, juin 1794), l'un dédié à l'arbre de la liberté et les deux autres célébrant l'existence de l'Éternel et de l'immortalité de l'âme. Ce thème semble beaucoup avoir inspiré Talairat puisqu'un nouvel hymne sur le sujet lui est attribué (BMIU Fonds Auvergne A35481 (7), Hymne à l'Etre suprême, juin 1794).

35.AD Cantal Fonds Delmas 27J206, Grou, Fiers habitants de la Montagne, 1793, Aurillac. 36.AD PDD. L6275, Anonyme, Chansons des voleurs, Juif errant, non daté. 37.AD PDD. L6275 (6), Anonyme, Je l'ai planté je l'ai vu naître, non daté. 
38. BMIU Fonds Paul Leblanc MS931.

39.Ibid.

40.Ibid.

41.AD PDD. L6275 (6).

42.AD PDD. L665 (1), L667 (10), L668 (37), L669 (1) ; AD Cantal 205F1, Fonds Delmas

27J206, 27J21 ; BMIU Fonds Auvergne A11294 (14), A11281 (27), A32309, A34934 (1 et 2),

Fonds patrimoine 70512 (2).

43.AD PDD. L666 (19), L668 (37), L670 ; BMIU Fonds Auvergne A11281 (27-28), A34934

(10-13), Fonds Paul Leblanc MS931.

44.AD PDD. L6385 ; AD Cantal Fonds Delmas 27J132 ; BMIU Fonds Auvergne A34934

(6-11).

45.BMIU Fonds Paul Leblanc MS931.

46.BMIU Fonds Auvergne A34934 (3).

47.BMIU Fonds Auvergne A32837.

48. AD Cantal Fonds Leymarie 6J27.

49.AD Cantal Fonds Leymarie 6J77.

50.Mona Ozouf, La fête révolutionnaire, Paris, Gallimard, 1988, p. 338.

51.L'extrait des registres de délibération de la commune de Jou, dans le Cantal, apparaît à ce titre tout à fait représentatif : «Ce jourd'hui trente ventôse an VI de la République une et indivisible [...] la cérémonie a commencé par le chant de la marseillaise, les vieillards se sont avancés au milieu de l'enceinte, ont réuni leur baguette et ont formé un fesseau qu'ils ont lié avec un ruban tricolore [...] et après la lecture il a été chanté des himnes patriotiques, après quoy somme retournés dans la maison commune» (AD Cantal L421/3). Ce procès-verbal serait passé de manière anodine à travers les mailles de la critique si l'on n'avait pas retrouvé le rapport de la fête en des termes identiques à Badalhac, Polminhac, Cartat, Pailherols, Raulhac et Thigeac.

52.Sur 65 procès-verbaux de fêtes dans le Puy-de-Dôme, 30 font référence à l'Hymne des Marseillais et 8 au Chant du Départ. Dans le Cantal, sur 16 procès-verbaux, le chant pour l'armée du Rhin est parodié à 7 reprises (ou simplement mentionné). Le dernier couplet Amour sacré de la patrie fait l'objet d'une attention particulière. On le pense capable d'électriser les âmes. Toutes les occasions semblent bonnes pour chanter l'hymne de Rouget de Lisle, de la fête de l'agriculture à celle des victoires. Le Chant du Départ, quoique très populaire dans sa diffusion, ne peut rivaliser.

53.Quel peut être l'impact populaire du couplet lyrique déclamé lors de la fête de l'Être suprême de Clermont-Ferrand dans lequel la libération de la France est symboliquement représentée par l'éclosion d'une rose grâce à la lumière de l'Éternel ? Cette emphase contraste avec la réalité quotidienne de la peur suscitée par l'ennemi et surtout par la Terreur. Quelle proportion de l'assistance est capable de saisir, à la seule lecture du couplet, l'image proposée par Nourry... et l'héritage poétique de Ronsard qu'il sollicite? (BMIU Fonds Auvergne A34934 (6)).

54.Francisque Mege, Chansons politiques et satiriques en Auvergne, Clermont-Ferrand, Mont Louis, 1886, p. 5.

55.Michelle Biget, Musique et Révolution française, Paris, Annales Littéraires de l'Université de Besançon, 1989, p. 97.

56.AD Cantal Fonds Delmas 27J132, anonyme, Avec les jeux dans le village, mai 1791, Cantal. Chaleureux hommage au nouveau représentant du clergé dans le Cantal, la chanson présente un prélat "vertueux », plein de « civisme » et de " courage »; un franc républicain dispensateur des idéaux de la Révolution qu'il s'agit d'honorer. 
57.AD Cantal L1099, Anonyme, Pamphlet contre l'évêque Thibault, juin 1791, Cantal. 58.Antoine de Baecque, La Caricature révolutionnaire, Paris, Presses du CNRS, 1988, p. 13. «Tout comme la chanson, le gros moine et le superstitieux réfractaire, l'aristocrate, l'émigré, le roi devenu porc, tous ces portraits caricaturaux, croqués dans le style allégorique ou la verve grotesque, offrent un commentaire à chaud de l'événement politique... »

59.AD PDD. L6275, Anonyme, Chanson des voleurs, Le juif errant, non daté. Saisie dans les papiers de deux religieuses que le conseil général de la commune de Champeix fait arrêter le 14 fructidor an II (31 août 1794), comme coupables d'avoir rétracté leurs serments, une composition met en scène un prêtre davantage séduit par sa solde que préoccupé par le salut de ses fidèles. Cette chanson au titre provocateur de Panégyrique des intrus sur l'air du Juif errant, prend le parti de Bonal, évêque de Clermont et député à l'Assemblée nationale qui se signale en toutes occasions comme un ardent défenseur de la religion et des droits du clergé et comme un adversaire résolu du parti patriote.

60.Martine Braconnier, op. cit., p. 215.

61.BMIU Clermont-Ferrand, A 34934 (3).

62.L'auteur, ayant une parfaite connaissance de l'hagiographie, reprend à son compte l'histoire populaire qui raconte que pendant son séjour dans un ermitage, un chien vient apporter chaque jour un pain au patron des pestiférés... Cette allusion fait écho à l'affaire de la relique du précieux sang. L'église Saint-Cerneuf de Billom abrite une relique très vénérée - un peu de sang du Christ apporté jadis par un chanoine de Palestine - que le représentant s'empresse de faire expertiser le 20 brumaire (10 novembre 1793) par un médecin du pays ; l'analyse conclut à l'imposture et permet à Couthon de remporter une nouvelle bataille sur le fanatisme.

63. Martine Braconnier, op. cit., note (60), p. 214. La croisade qu'il mène contre la superstition, et une politique plus globale d'éradication de l'Église, l'encourage à prendre un arrêté le 24 brumaire connu sous le nom d'arrêté sur le dépouillement des églises. Les quatre séries d'articles visent entre autres à supprimer les titres religieux, à détruire les objets du culte et symboles religieux, à confisquer l'instruction au profit des sociétés populaires...

64.AD Cantal Fonds Leymarie 6j77, Delolm Labaudie, Couplets sur la prise de Toulon, Je ne forme point de désir, non daté, Aurillac.

65.L'utilisation de la lâcheté ennemie a le don de valoriser les courageux citoyens français et tend à placer le théâtre de la guerre aux frontières de l'Auvergne : l'ennemi contre-révolutionnaire est susceptible de tramer ses méfaits partout. Ce sentiment ne transparaît dans la chanson auvergnate que tardivement...

66.Sophie Wahnich, L'impossible citoyen : l'étranger dans le discours de la Révolution française, Paris, Albin Michel, 1997, p. 206.

67.Philippe Bourdin, op. cit., p. 173.

68.Les incidents de Rastadt tendent à mobiliser la République civilisée face à la barbarie autrichienne. Romeuf participe à cet effort par un hymne de son inspiration sur l'air Soldat le bal va se rouvrir composé pour la fête funéraire du 20 prairial an VII (8 juin 1799) dans lequel l'auteur accorde deux temps à cette cérémonie funéraire : le recueillement pour la paix de l'âme des plénipotentiaires et la haine envers ceux qui ont commis de telles atrocités, le but étant bien entendu de raviver la flamme patriotique. Plus véhéments encore sont les couplets clermontois sur la mort de Bonnier, ancien conseiller à la Cour des aides de Montpellier, et Roberjot, curé défroqué, envoyés à Rastadt pour négocier la paix (BMIU Fonds Auvergne A34904, 
Anonyme, Plaignez ce vieillard éperdu, 1799) dans lesquels l'auteur lance un appel à témoin aux autres nations afin de montrer que cet événement n'a jamais eu de précédents historiques. L'Autriche se met au ban des nations pour sa cruauté. 69.La région a certes dû beaucoup souscrire à cet impôt de sang ; voir sur la question Bruno Ciotti, Du volontariat au conscrit : les levées militaires dans le département du Puy-deDôme sous la Révolution (1791-an VII) ; recrutement et population, Thèse d'État, Lyon, Université Lumière, 1995.

70.AD PDD. L670, Armand, Chant du départ des bataillons du Puy-de-Dôme, Chant du départ, décembre 1799, Clermont-Ferrand.

71. Claude Mossé, L'Antiquité et la Révolution, Paris, Albin Michel, 1989, p. 23.

72.Desfeorges ne manque pas l'opportunité de rendre un vibrant hommage à l'agriculteur et au soldat dès la fête de l'agriculture du 10 messidor an IV (29 juin 1796) (AD PDD. L6385, Desfeorges, Hymne à l'agriculture, Avec les jeux dans le village, juin 1795). 73.Les chansons contre-révolutionnaires rencontrées sont versées dans la série L, la plupart du temps au chapitre des lettres interceptées.

74.AD Cantal Fonds Leymarie 6J11, Anonyme, Couplets de l'époque de la Terreur, 1794. 75.BMIU Fonds Paul Leblanc MS931, Anonyme, Cri de mort contre les conspirateurs et les hommes de sang, Marseillaise, après thermidor an II, Brivadois.

76.AD Cantal EDEP1500 419, Lakairie, À la paix, Dans le cœur d'une cruelle, novembre 1797, Aurillac.

77.Jean-Louis Jam dans Révolution et « vandalisme " révolutionnaire, actes du colloque de Clermont-Ferrand, recueillis et présentés par Simon Bernard GRIFFITHS, Marie-Claude CHEMIN et Jean EHRARD, organisé par l'Université Blaise Pascal, Clermont-Ferrand, Faculté des Lettres et Sciences Humaines, CRRR, Paris, Universitas, 1992, p. 290.

\section{RÉSUMÉS}

On n'a peut-être jamais autant chanté qu'à l'époque révolutionnaire; cette décennie mouvementée permet à la multitude de suivre son penchant naturel. On chante la Révolution dans les rues, dans les salons, dans les fêtes, sur les théâtres. Si Paris reste incontestablement le lieu de prédilection des chansonniers, la chanson se diffuse également en Auvergne. Ce panorama peut paraître subjectif avec le recul du temps, mais les chansons, écrites dans la fièvre du moment, sont le reflet de la sensibilité auvergnate. Eloignée des grands théâtres d'opérations militaires et de guerre civile, exempte de mouvements politiques violents et de troubles religieux aigus - la Terreur y est plus modérée et la déchristianisation larvée - l'Auvergne ${ }^{1}$ ne présente à l'égard des péripéties révolutionnaires ni enthousiasme prononcé ni hostilité systématique. Si bien qu'à la marche précipitée de la Révolution parisienne correspond ici un rythme plus large : l'onde s'amplifie et se calme en s'éloignant de son épicentre. L'enjeu pour le nouveau régime, par le biais de ses administrateurs, de ses représentants ou de ses ministres, consiste à faire adhérer la population aux idéaux républicains. Pour cela, l'art, et en particulier le formidable vecteur de propagande qu'incarne la musique, est ballotté entre impulsion et dirigisme. La chanson se veut également moyen d'éducation, de sensibilisation aux idées du siècle d'une population analphabète à $50 \%$; la musique monte sur scène. Mais à trop vouloir enrégimenter l'activité chansonnière, les administrateurs locaux ne parviennent guère à susciter l'enthousiasme d'une 
population davantage préoccupée par les problèmes de ravitaillement et de conscription que la Révolution génère. À partir de l'an II (1794), les pédagogues se détournent de leur public, la musique tend à perdre sa principale raison d'être... l'engouement populaire.

La première partie, état des lieux des sources disponibles, rappelle les différents modes de diffusion des productions auvergnates, la prééminence patente des cités en tant que principaux foyers d'Orphée; la seconde s'attache à l'élaboration de la figure du chansonnier auvergnat, personnage issu de la petite bourgeoisie à la solde du régime; la troisième partie établit un lien entre le contexte régional brûlant et les hymnes produits.

Singing as a revolutionary "weapon " and echoing the society in Auvergne. French people probably have never sung as much as during the Revolution. People sing in the streets, in the salons, in the fairs or in the theatres. The songs written during this period reflects the sensitivity of the people living in Auvergne. Away from military battles, from the civil war, free from violent political unrests and religious troubles, they are neither deeply enthusiastic nor systematically hostile to the turns taken by the revolution. The rhythm of the events is slower than in Paris. By the means of its ministers, the goal of the new regime consists in making the French people adhere the republican ideals. That's why art, and particularly music, used as a means of propaganda, is tossed between impulse and the government's demand. Moreover songs are a way to educate, to make an illiterate population sensitive to the ideas of this century. Music is on the stage. But local administrators don't really manage to fill with enthusiasm a population worried about getting fresh supplies and concerned about conscription. From 1794, teachers turn away from their public and music tends to lose its popularity. The first part, which is an inventory of the available sources, deals with the different ways of spreading music in Auvergne, cities being the main Orpheus centres. The second part focuses on the chansonniers belonging to the middleclass and being in the pay of the regime. Then, the third part establishes a link between the burning regional context and the produced hymns.

\section{INDEX}

Mots-clés : Auvergne, hymne, chanson, propagande, paroliers-administrateurs.

\section{AUTEUR}

\section{FRÉDÉRIC DERNE}

Doctorant. Université Blaise-Pascal (Clermont-Ferrand II), Centre d'Histoire « Espaces et Cultures », Maison de la recherche, 4 rue Ledru, 63057 Clermont-Ferrand cedex 1 